\title{
Wear-Related Phenomena in Advanced Materials
}

\author{
Alexander Tsouknidas, ${ }^{1}$ Luca Settineri, ${ }^{2}$ Pedro Arrazola, ${ }^{3}$ and Nikolaos Michailidis ${ }^{4}$ \\ ${ }^{1}$ Laboratory for Machine Tools and Manufacturing Engineering, Mechanical Engineering Department, \\ Aristotle University of Thessaloniki, 54124 Thessaloniki, Greece \\ ${ }^{2}$ Department of Production Systems and Business Economics, Polytechnic University of Turin, 10129 Torino, Italy \\ ${ }^{3}$ Mechanical and Manufacturing Department, Mondragon University, 20500 Arrasate-Mondragón, Spain \\ ${ }^{4}$ Physical Metallurgy Laboratory, Mechanical Engineering Department, Aristotle University of Thessaloniki, P.O. BOX 490, \\ 54124 Thessaloniki, Greece \\ Correspondence should be addressed to Nikolaos Michailidis, nmichail@eng.auth.gr
}

Received 19 December 2011; Accepted 19 December 2011

Copyright (C) 2011 Alexander Tsouknidas et al. This is an open access article distributed under the Creative Commons Attribution License, which permits unrestricted use, distribution, and reproduction in any medium, provided the original work is properly cited.

\section{Aims and Scope of the Issue}

The title of this editorial reflects an effort in creating a unique research forum, to harbor fundamental as well as review articles, meeting the forefront of this scientific field. The Guest Editors sought to attract articles on friction, wear, surface properties, and the development of sufficient procedures, to arrest wear progression as well as to indicate mechanisms to prolong the effectiveness of the implicated materials.

In these terms it was exciting and rewarding to embrace related papers of scientists from around the world. The concept of wear-related phenomena in advanced materials, along with a short introduction on the subjects, addressed by the accepted papers, will be presented in the following paragraphs.

\section{Introduction}

Wear has, by its very nature, always dominated the interest of engineers and physicists, as it represents a material property of enormous practical importance. Even though it is a multidisciplinary phenomenon based on simultaneously occurring mechanical, physical, and chemical processes, recent advances in sophisticated material design and development and characterization techniques have sustained methodologies to comprehend and predict wear progression. The capacity to systematically and effectively describe wear development is a perspective of great importance to engineers who use materials and mechanical components or are directly involved with their manufacturing processes.

\section{Wear in Advanced Materials}

Milling is a characteristic manufacturing process in which wear exerts a dominating effect on both product quality and cost. There exists a consensus throughout the literature that high-performance cutting of difficult-to-machine materials strongly depends on the engagement parameters as well as on the tool and workpiece materials, and thus analytical-experimental optimization procedures may lead to drastic enhancement of the tool life, as discussed in the paper entitled "Characterization of tool wear in highspeed milling of hardened powder metallurgical steels." This is particularly important when processing advanced materials like $\mathrm{Ti}$ - or Ni-based alloys. Over the past years, the related manufacturing procedures have arisen from their period of infancy and gained tremendous importance in several industrial sectors, that is, aerospace. It has been sustained that novel heuristic optimization procedures can be successfully applied during the integration of milling strategies, for such materials, providing high removal rates while maintaining acceptable production costs, as evidenced in the paper entitled "Integrated approach for a knowledgebased process layout for simultaneous 5-axismilling of advanced materials."

Alternative methods, like additive manufacturing, have been recently introduced for the production of functionallygraded, high-end materials. Even though this eases the production of complex geometries, these technologies produce components prone to several degeneration scenarios and thus, it is pivotal to consider a variety of production and application-based parameters, as showen in the paper 
entitled "Friction induced wear of rapid prototyping generated materials: A review."

Antifriction-based materials and components are predominantly developed, based on application-specific requirements. Powder metallurgy is a key enabling technique in this direction, facilitating, among others, the production of self-lubricating components (i.e., bearings). Recent investigations on iron-based alloys indicated a strong enslavement of the mechanical and tribotechnical properties of the final product to the production parameters, which must therefore be carefully considered, as proven in the papers entitled "Development of new PM iron base materials for self-lubricating bearings." and "Model of fracture, friction, and wear phenomena of porous iron." Aluminum-based $\mathrm{TiB}_{2}$-reinforced composites are widely considered as an alternative to ferrous antifriction components. Late studies revealed that the wear behavior of these composites is comparable to the one of gray cast iron parts, as exhibiled in the paper entitled "Wear behavior and its correlation with mechanical properties of $\mathrm{Ti}_{2}$ reinforced aluminium-based composites." The high cost of aluminum composites can be decreased through recovery procedures of dross, produced in large quantities "A characterization method for Al recovery from dross based on compression at elevated temperatures." Related procedures have been optimized, and the purity of the recovered material is considered as acceptable for the production of high-added-value products.

\title{
Epilogue
}

Our sincere hope and wish is that "Advances in Tribology" will continue to address the multidisciplinary phenomena reflected in the wear development of advanced materials, and we commit ourselves to support this effort in our best capacity.

\author{
Alexander Tsouknidas \\ Luca Settineri \\ Pedro Arrazola \\ Nikolaos Michailidis
}



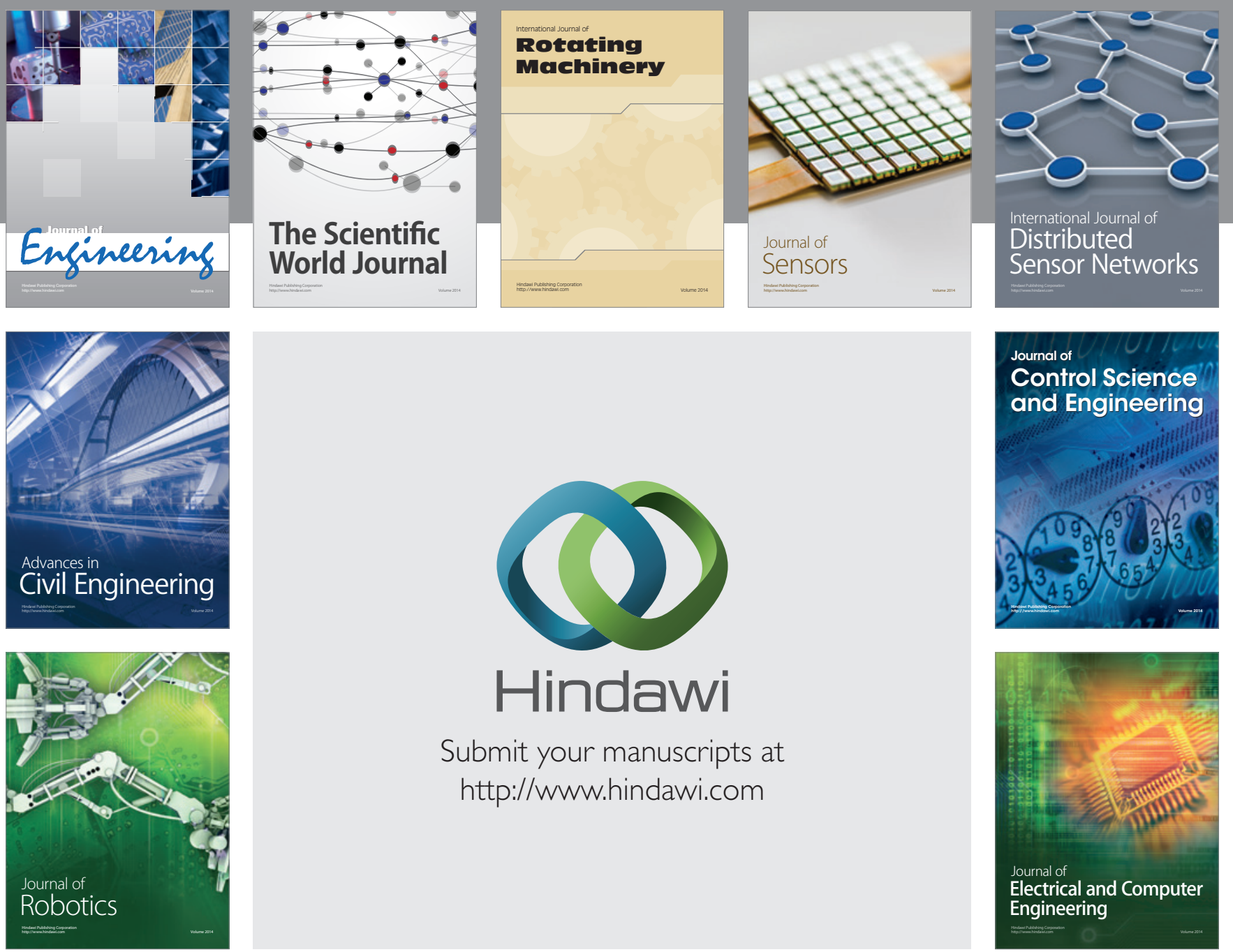

Submit your manuscripts at

http://www.hindawi.com
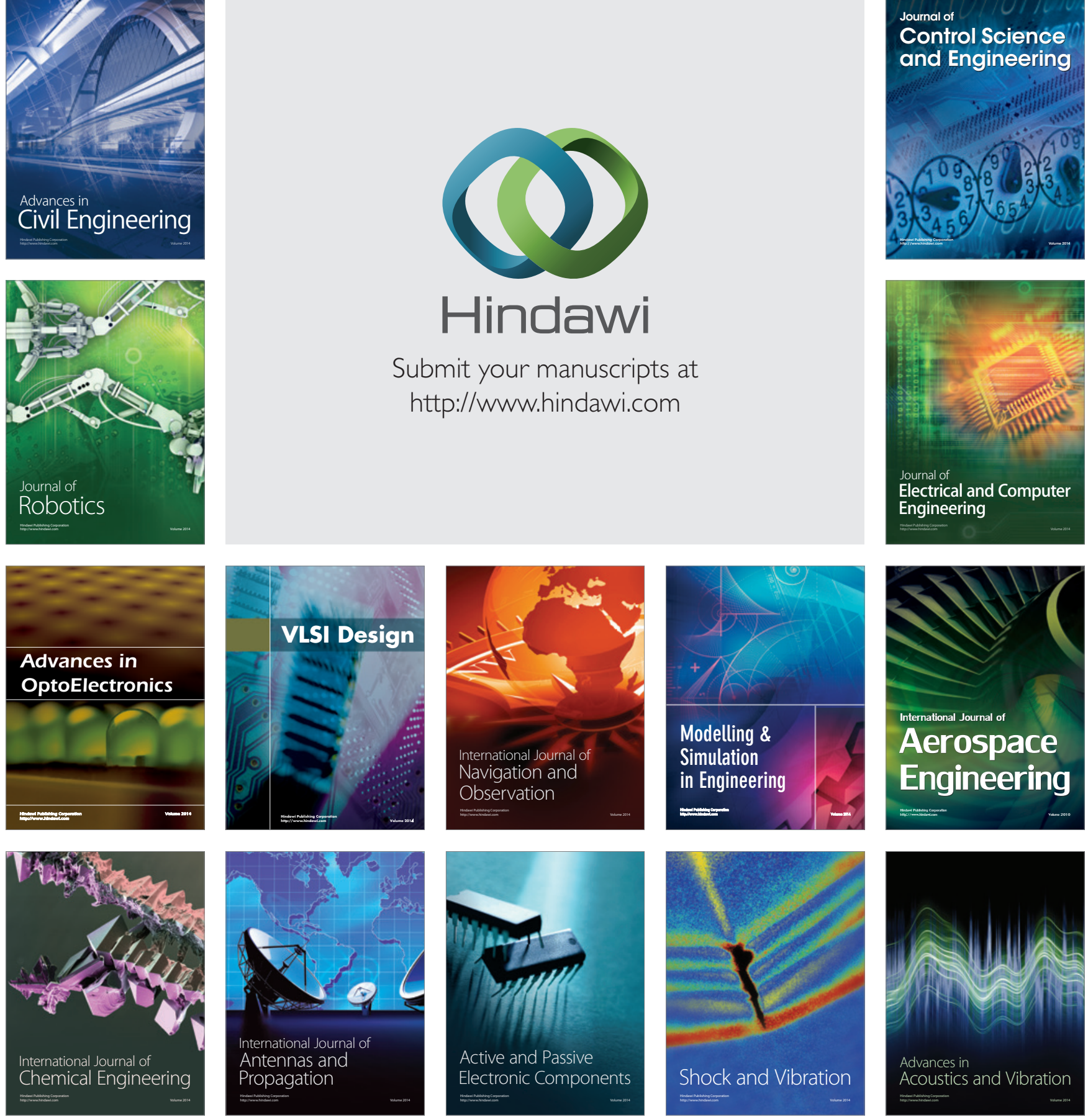